\title{
Artificial intelligence in nuclear cardiology: Preparing for the fifth industrial revolution
}

\author{
Ernest V. Garcia, PhD ${ }^{\mathrm{a}}$ \\ a Department of Radiology and Imaging Sciences, Emory University School of Medicine, Atlanta, \\ GA
}

Received May 5, 2021; accepted May 5, 2021

doi: $10.1007 / \mathrm{s} 12350-021-02671-1$

\begin{abstract}
"The past is but the beginning of a beginning, and all that is or has been is but the twilight of the dawn." HG Wells, The Discovery of the Future (1913)
\end{abstract}

Seldom does a novel technology come along that dramatically transforms production, and when it does, the term that is used to identify it is an Industrial Revolution (IR). Whereas the $3^{\text {rd }}$ IR dealt with computers and the internet for automation and globalization and the $4^{\text {th }}$ IR with artificial intelligence (AI) and robotics for digitization, the $5^{\text {th }}$ IR is being recognized as the rise of $\mathrm{AI}^{1}$ for personalization. ${ }^{2}$ Personalization has, at a minimum, two purposes. First, it relates to how products and services are designed, manufactured, and deployed. In Nuclear Cardiology (NC), one example is patient-centered imaging ${ }^{3}$ but also analysis, diagnosis, prognosis, reporting, and treatment guidance that is patient-specific. Second, personalization also relates to the integration and unique partnership between AI and humans in the workplace without fearing AI's potential attributes of non-bias, data-driven accuracy for better informed decisions. ${ }^{1}$

This article is a clarion call to our field to prepare for the magnitude and speed of the AI-tsunami that is coming. Our intuition as to how significant and quick these changes will come fail us for a number of reasons. Our intuition is biased by our experience of the continuous progress in computer technology and the internet during the last 50 years of the $3^{\text {rd }}$ IR as well as in robotics and AI in the last 30-50 years of the $4^{\text {th }}$ IR. Our intuition is also biased by many years that it has taken to

\footnotetext{
Reprint requests: Ernest V. Garcia, PhD, Department of Radiology and Imaging Sciences, Emory University School of Medicine, 101 Woodruff Circle, Room 1203, Atlanta, GA 30322; ernest.garcia@emory.edu

J Nucl Cardiol 2021;28:1199-202.

1071-3581/ $\$ 34.00$

Copyright (c) 2021 American Society of Nuclear Cardiology.
}

implement NC-centric developments such as SPECT attenuation correction, solid state cameras, and PET flow measurements. The much faster speed of the rise of the AI-tsunami in the $5^{\text {th }}$ IR is due to its capability to ride a global technology wave ${ }^{4}$ that includes the major accomplishments of the previous IRs plus major developments in the Cloud, internet speed/bandwidth, big data, natural language understanding, and open-sourced machine learning (ML) including deep learning ${ }^{5}$ (DL). Moreover, these speeds of AI implementations are accelerated by major financial investments in the technology. In 2019 alone, AI companies attracted investments of $\$ 40$ billion globally, $\$ 25$ billion in the USA. $^{6}$

\section{IMPLICATIONS FOR NUCLEAR CARDIOLOGY}

Many significant AI developments have already been reported in nuclear cardiology. These developments include using DL for reducing the needed injected dose and acquisition time in MPI acquisitions ${ }^{7,8}$ also due to DL improvements in image reconstruction and filtering, ${ }^{9}$ SPECT attenuation correction using DL without need for transmission images, ${ }^{10} \mathrm{DL}$ and ML use for feature extraction to define myocardial $\mathrm{LV}$ borders ${ }^{11}$ for functional measurements and improved detection of the LV valve ${ }^{12}$ plane and AI, ML, and DL implementations for MPI diagnosis, ${ }^{13-15}$ prognosis, ${ }^{16,17}$ and structured reporting. ${ }^{13}$ Although some have, ${ }^{13}$ most of these applications have yet to make it to widespread commercial distribution due to the recency of their developments, most reported in 2020. Excellent, recent comprehensive articles ${ }^{18,19}$ provide a more complete review of the AI applications that have arrived in cardiovascular imaging.

The remainder of this article suggests how the various NC stakeholders should prepare for the $5^{\text {th }}$ IR to exploit the opportunities that it brings and avoid potential threats. Similar perspectives have been offered for all healthcare. ${ }^{20}$ 


\section{IMPLICATIONS FOR INSTITUTIONS}

Perhaps the most important role in preparing for the $5^{\text {th }}$ IR lies with the clinical institutions that generate data, including both actual images and clinical variables. This is because the ML and DL algorithms are mostly driven by data rather than by the sophistication or cleverness of the scientific code. In fact, the majority of the code needed for this AI may be obtained for free as open-source code and it is the data that trains the algorithms and where the value lies. Thus the saying"Data is the new oil."' Following this analogy, it may be argued that most healthcare systems are sitting on top of major oil deposits and do not even know it.

Some institutions do know the newfound value of clinical data and are exploiting it. Sloan-Kettering Cancer Center is providing data to Paige AI in the form of 25 million image sets of patient tissue slides and pathologists findings to develop AI algorithms to assist in cancer diagnosis. ${ }^{21,22}$ Allegedly, the center provides exclusive use of these data for a $9 \%$ equity stake in the company. In another deal, St. Louis, Missouri-based Ascension, a Catholic chain of 2600 hospitals, agreed to share data with Google in a project with the code name "Project Nightingale.,"22,23 The reported shared data ${ }^{22}$ include radiology scans, laboratory tests, hospitalization records, medical conditions, and patient's personal information. These deals and many others follow the footsteps of IBM which in 2016 purchased Truven Health Analytics for $\$ 2.6$ billion for their healthcare data on tens of millions of medical records and years of health insurance data. ${ }^{22}$

Legal and ethical issues aside, which will be discussed below, these major financial transactions form part of the foundation for the fast-approaching AItsunami. Just like oil needs to be extracted and refined to be useful, institutions would benefit from having personnel to curate their clinical data and to have systems that can transparently access it. The purpose to this ready access to carefully curated, annotated clinical data is twofold. Access is both a source of valuable data for AI training but also more importantly to be able to benefit from the algorithms developed by others including academic investigators and industry.

\section{IMPLICATIONS FOR PROFESSIONAL SOCIETIES}

Professional societies have a role, perhaps a responsibility, in becoming a catalyst to expedite the deployment of the $5^{\text {th }}$ IR in our field. Just as is commonly done by computer societies, healthcare societies can provide a major service by being one source of the needed big databases from contributions by many institutions for training algorithms. Although in the past this has created legal and financial concerns, the time is now to resolve it. Creative business models may be developed to reward all concerns and move the field forward. These databases, by their annotated nature, would also provide a source of validation, a valuable asset for AI algorithms development and regulatory clearance. Finally, by being an integral part of the development and validation process, societies could become more relevant by fast tracking the new AI algorithms into their imaging guidelines, vastly reducing the many years that it now takes from innovation to recommendation.

\section{REGULATORY IMPLICATIONS FOR THE DEVICE AND RADIOPHARMACEUTICAL INDUSTRIES}

Initially, there was concern as to how to regulate AI algorithms that came in the form of a black box, i.e., with no description as to how the algorithm reached conclusions or made decisions as these algorithms are mostly a set of training weights. The FDA, realizing that the coming AI-tsunami has the potential to transform healthcare has adapted its vision as described in their recently published action plan ${ }^{24}$ : “ "...with appropriately tailored total product lifecycle-based regulatory oversight, AI/ML-based Software as a Medical Device (SaMD) will deliver safe and effective software functionality that improves the quality of care that patients receive." This follows the FDA's publication ${ }^{25}$ on a proposed regulatory framework describing their foundation for a potential approach to premarket review for AI- and ML-driven software modifications. This action plan recognizes and embraces the iterative improvement power of $\mathrm{AI} / \mathrm{ML} / \mathrm{DL}$ allowing a post-market mechanism for continuously improving the algorithms as new data is available. The process would be constrained to specific procedures and meeting certain target accuracy thresholds. These FDA actions are major encouragements for the medical device industry to fully utilize all the benefits of the $5^{\text {th }}$ IR.

A similar AI vision and concessions are also required from the radiopharmaceutical division of the FDA. Devices and imaging radiopharmaceuticals have a symbiotic relationship in that the radiopharmaceuticals' FDA clinical trials follow a rigorous imaging protocol that then becomes part of the product's package insert. To change the package insert, usually it requires another expensive clinical trial. Deviation from the package insert may be done under the practice of medicine but may not be promoted by either the pharmaceutical or the device company. A simple example is when devices are improved, such as DL-assisted reconstruction to reduce the needed injected dose, and the new required dose falls below the lower limit listed in the radiopharmaceutical 
package insert. Even though this could result in a reduction of radiation to the patient and a potential financial saving to the healthcare industry, there is no present regulatory framework for its implementation. Coalitions of radiopharmaceutical companies should prioritize discussions with the FDA to implement these frameworks. Consideration should be given to both retrospective and prospective trials. In this context, retrospective trials are FDA trials that are completed resulting in FDA approval of the radiopharmaceutical imaging agent and now there are data to modify the package insert based on algorithm improvements applied to the same original data. For prospective trials that companies are designing now, strong consideration should be given to permanently storing the original imaging data in its most elementary acquired raw form so it may be reused each time new algorithms improve efficiency but affect the original imaging protocols. In this scenario, FDA should be able to accept these results as those coming from a "new" clinical trial that allows the change in package insert.

\section{LEGAL, ETHICAL IMPLICATIONS}

As usual, legal and ethical norms woefully drag behind technological developments. This is even truer for the AI-tsunami which is the $5^{\text {th }}$ IR and because the legal and ethical issues are intertwined. There are two main legal implications: who owns the patients' data, for example their MPI studies, and who is liable for using the results of an AI algorithm. Legally in the USA, clinical data are considered the property of the provider organization and can be sold by the organization as long as patient access and privacy protection requirements are met. 22 "The Immortal Life of Henrietta Lacks"' is a book and film about a true story of the case that helped provide the legal precedence to the ownership court decision. $^{26}$

The question of liability for clinical malpractice in the USA is always dependent on the judgment of jurors and thus if sued, for whatever reason, one needs a defense. Perhaps unexpectedly, a recent study ${ }^{27}$ sampling a representative set of 2000 adults presented with four scenarios in which the AI system provided treatment recommendations to a physician indicated that physicians who receive advice from an AI system to provide standard care can reduce their risk of liability by accepting, rather than rejecting, that advice, all else being equal. However, when an AI system recommends nonstandard care, there is no similar shielding effect of rejecting that advice and so providing standard care. These investigators concluded that tort law system is unlikely to undermine the use of AI precision medicine tools and may even encourage the use of these tools. ${ }^{27}$
Ethicists have not reached consensus as to ethical norms for using and sharing clinical imaging data for AI. It has been proposed, and generally accepted, that all who participate in the health care system, including patients, have a moral obligation to contribute to improving that system ${ }^{28}$ and thus to allow the use of their imaging studies and clinical data for the development of AI algorithms. What is contested is whether it is ethical for this valuable clinical data to be sold or should it become public to benefit future patients. ${ }^{22}$ Since it is legal to sell the data as a significant revenue-producing component of a healthcare system, history dictates that the data will not be given away for free. A possible exception is a scenario where multiple institutions participate voluntarily, and to a limited degree, to create a big database for the benefit of the field.

\section{SECURITY IMPLICATIONS}

It is inevitable that the super tools and big data warehouses that are coming with the $5^{\text {th }}$ IR will be used by hackers for nefarious purposes in ways yet to be imagined. Institutions should have a well-designed backup plan that includes, at a minimum, remote redundant storage with non-internet access for all clinical data.

Will there ever be a solution to dealing with hackers? Perhaps that will be the focus of the $6^{\text {th }}$ Industrial Revolution where quantum computing will be used for decrypting any nefariously encrypted files but more importantly where quantum entanglement will be used to protect all data access.

\section{Disclosure}

Dr. Ernest Garcia receives royalties from the sale of the Emory Cardiac Toolbox and has equity positions with Syntermed, Inc. The terms of these arrangements have been reviewed and approved by Emory University in accordance with its conflict of interest policies.

\section{References}

1. Bridgman R, Are you ready for the 5th industrial revolution? (2019) https://www.recruitment-international.co.uk/blog/2019/10/ are-you-ready-for-the-5th-industrial-revolution. Accessed May 3, 2021.

2. The Fifth Industrial revolution and how it will change the business landscape, REGENESYS (2020). https://insights.regenesys.net/th e-fifth-industrial-revolution-5ir/ Accessed May 3, 2021.

3. DePuey EG, Mahmarian JJ, Miller TD, et al. Patient-centered imaging. J Nucl Cardiol 2012;19:185-215. https://doi.org/10.1007/ s12350-012-9523-z. 
4. Garcia E. My Ride to the Cloud. TEDx Talks, (2017). Accessed May 3, 2021. https://www.youtube.com/watch?v=Tiwcc2opa04

5. LeCun Y, Bengio Y, Hinton G. Deep learning. Nature 2015;521:436-44.

6. Arnold Z. What investment trends reveal about the global AI landscape (2020). https://www.brookings.edu/techstream/what-in vestment-trends-reveal-about-the-global-ai-landscape Accessed May 3, 2021.

7. Song C, Yang Y, Wernick MN, et al. Low-dose cardiac-gated SPECT studies using a residual convolutional neural network. 2019 IEEE 16th International Symposium on Biomedical Imaging (ISBI 2019), 2019, 653-656.

8. Shiri I, Sabet KA, Arabi H, et al. Standard SPECT myocardial perfusion estimation from half-time acquisitions using deep convolutional residual neural networks. J Nucl Cardiol 2020. https://d oi.org/10.1007/s12350-020-02119-y.

9. Ramon AJ, Yang Y, Pretorius PH, et al. Improving diagnostic accuracy in low-dose SPECT myocardial perfusion imaging with convolution denoising networks. IEEE Trans Med Imaging 2020;39:2893-903.

10. Shi L, Onofrey JA, Liu H, et al. Deep learning-based attenuation map generation for myocardial perfusion SPECT. Eur J Nucl Med Mol Imaging 2020;47:2383-95. https://doi.org/10.1007/s00259-0 20-04746-6.

11. Wang T, Lei Y, Tang H, et al. A learning-based automatic segmentation and quantification method of left ventricle in gated myocardial perfusion SPECT imaging: A feasibility study. J Nucl Cardiol 2020;27:976-87.

12. Betancur J, Rubeaux M, Fuchs TA, et al. Automatic valve plane localization in myocardial perfusion SPECT/CT by machine learning: Anatomic and clinical validations. J Nucl Med 2017;58:961-7.

13. Garcia EV, Klein JL, Moncayo V, et al. Diagnostic performance of an artificial intelligence-driven cardiac-structured reporting system for myocardial perfusion SPECT imaging. J. Nucl. Cardiol 2020;27:1652-64. https://doi.org/10.1007/s12350-018-1432-3.

14. Betancur J, Hu LH, Commandeur F, et al. Deep learning analysis of upright-supine high-efficiency SPECT myocardial perfusion imaging for prediction of coronary artery disease: A multicenter study. J Nucl Med 2019;60:664-70.

15. Juarez-Orozco LE, Knol RJJ, Sanches-Catasus CA, et al. Machine learning in the integration of simple variables for identifying patients with myocardial ischemia. J Nucl Cardiol 2020;27:14755.

16. Alonso DH, Wernick MN, Yang Y, et al. Prediction of cardiac death after adenosine myocardial perfusion SPECT based on machine learning. J Nucl Cardiol 2019;26:1746-54.
17. Betancur J, Otaki Y, Motwani M, et al. Prognostic value of combined clinical and myocardial perfusion imaging data using machine learning. JACC Cardiovasc Imaging 2018;11:1000-9.

18. Lin A, Kolossváry M, Išgum S, Maurovich-Horvat $\mathrm{P}$, et al. Artificial intelligence: Improving the efficiency of cardiovascular imaging. Exp Rev Med Devices 2020;17(6):565-77. https://doi. org/10.1080/17434440.2020.1777855.

19. Slart RHJA, Williams MC, Juarez-Orozco LE, et al. Position paper of the EACVI and EANM on artificial intelligence applications in multimodality cardiovascular imaging using SPECT/CT, PET/CT, and cardiac CT. Eur J Nucl Med Mol Imaging 2021. https://doi. org/10.1007/s00259-021-05341-z.

20. Sung J, Stewart CL, Freedman B. Artificial intelligence in health care: Preparing for the fifth Industrial Revolution. MJA 2020. h ttps://doi.org/10.5694/mja2.50755.

21. Ornstein C, Thomas K. Sloan Kettering's cozy deal with start-up ignites a new uproar. New York Times. https://www.nytimes.com/ 2018/09/20/health/memorial-sloan-kettering-cancer-paige-ai.html. Published September 20, 2018. Accessed May 5, 2021.

22. Larson DB, Magnus DC, Lungren MP, Shah NH, Langlotz CP. Ethics of using and sharing clinical imaging data for artificial intelligence: A proposed framework. Radiology 2020;295:675-82.

23. Copeland R. Google's 'Project Nightingale' gathers personal health data on millions of Americans. Wall Street Journal. https:// www.wsj.com/articles/google-s-secret-project-nightingale-gather s-personal-health-data-on-millionsof-americans-11573496790?mo d=article_inline. Published November 11, 2019. Accessed May 5, 2021.

24. Artificial Intelligence/Machine Learning (AI/ML)-Based software as a medical device (SaMD) Action Plan (2021) https://www.fda.g ov/media/145022/download Accessed May 5, 2021

25. Proposed Regulatory Framework for Modifications to Artificial Intelligence/Machine Learning (AI/ML)-Based Software as a Medical Device (SaMD) - Discussion Paper and Request for Feedback. (2019) https://www.fda.gov/media/122535/download Accessed May 5, 2021

26. Skloot R. The Immortal Life of Henrietta Lacks. New York, NY: Penguin Random House; 2010.

27. Tobia K, Nielsen A, Stremitzer S. When does physician use of AI increase liability? J Nucl Med 2021;62:17-21. https://doi.org/10. 2967/jnumed.120.256032.

28. Faden RR, Kass NE, Goodman SN, Pronovost P, Tunis S, Beauchamp TL. An ethics framework for a learning health care system: A departure from traditional research ethics and clinical ethics. Hastings Cent Rep 2013;43:S16-27.

Publisher's Note Springer Nature remains neutral with regard to jurisdictional claims in published maps and institutional affiliations. 